(2) Open Access Full Text Article

\title{
Inhibiting eEF-2 kinase-mediated autophagy enhanced the cytocidal effect of AKT inhibitor on human nasopharyngeal carcinoma
}

This article was published in the following Dove Press journal: Drug Design, Development and Therapy

\author{
Yuan-yuan Zhao ${ }^{1, *}$ \\ Ying Tian ${ }^{1, *}$ \\ Lin Liu',2,* \\ Jian-hua Zhan' \\ Xue Hou' \\ Xi Chen' \\ Ting Zhou' \\ Yan Huang' \\ Li Zhang'
}

'Department of Medical Oncology, Sun Yat-sen University Cancer Center; State Key Laboratory of Oncology in South China; Collaborative Innovation Center for Cancer Medicine;

Guangdong Key Laboratory of Nasopharyngeal Carcinoma Diagnosis and Therapy, Guangzhou, China; ${ }^{2}$ Zhaoqing Medical College, Zhaoqing, Guangdong Province, People's

Republic of China

*These authors contributed equally to this work

\begin{abstract}
Aim: Our previous research showed that AKT inactivation via small molecule inhibitors did not induce significant apoptosis, but rather markedly increased autophagy in nasopharyngeal carcinoma (NPC). The purpose of the current study was to determine whether autophagy inhibition can enhance the anticancer efficacy of an AKT inhibitor (MK-2206).

Materials and methods: NPC cell lines CNE-2 (Epstein-Barr virus negative) and C666-1 (Epstein-Barr virus positive) were used to conduct the research. Autophagy induction effects were evaluated via Western blotting. Eukaryotic elongation factor-2 (eEF-2) kinase was specifically and stably knocked down using shRNA. The growth and proliferation of the cells were assessed by Cell Counting Kit- 8 . In CNE- 2 xenograft tumors, the antitumor effects of an AKT inhibitor (MK-2206) combined with an eEF-2 kinase inhibitor (NH125) were tested.

Results: MK-2206 induced eEF-2 kinase-dependent autophagy in NPC cell lines. Knockdown of eEF-2 kinase using shRNA blunted the autophagy activated by MK-2206. Compared with treatment with MK-2206 alone, shRNA or NH125 suppressed eEF-2 kinase and increased the growth-inhibitory effect of MK-2206 on the human NPC cell lines. The synergistic effects of eEF-2 kinase inhibition and MK-2206 were similar to those of the combination of hydroxychloroquine and MK-2206. Moreover, NH125 showed good synergistic effects with MK-2206 in vivo.

Conclusion: eEF-2 kinase-mediated autophagy induced by AKT inhibition played a protective role in NPC cells. Inhibition of eEF-2 kinase may be an effective method for increasing the efficacy of an AKT inhibitor such as MK-2206 in NPC.
\end{abstract}

Keywords: nasopharyngeal carcinoma, NPC, AKT, eEF-2 kinase, autophagy

\section{Introduction}

Nasopharyngeal carcinoma (NPC) is a cancer that arises from the epithelium of nasopharynx. NPC is rare throughout most of the world, but frequently occurs in certain geographic areas, such as Southeast Asia and southern China with an incidence rate varying from 20 to 50 cases per 100,000 people. ${ }^{1,2}$ Although early-stage NPC can be cured by radiotherapy, a significant number of patients still show local recurrence and distant metastases, decreasing the 5-year overall survival rate from $100 \%$ to $86.1 \%$ for Stage I-II disease to $50.7 \%$ for Stage IV disease. ${ }^{3}$ In the metastatic setting, despite the relative chemosensitivity of NPC, resistance to chemotherapy inevitably develops with median overall survival generally $<24$ months. ${ }^{4}$ Therefore, it is necessary to gain a better understanding of the molecular mechanisms underlying therapeutic failure and to develop an effective strategy for NPC therapy. ${ }^{5}$

AKT, a serine-threonine kinase, plays a central role in the PI3K/AKT/mammalian target of rapamycin (mTOR) signaling pathway. ${ }^{6}$ The PI3K/AKT pathway is emerging 
as possibly one of the most important pathways in head and neck squamous cancers and is constitutively activated in the NPC tissue. ${ }^{7,8}$ However, our previous preclinical study demonstrated that AKT inhibition by MK-2206 had a modest antitumor effect on NPC both in vitro and in vivo. ${ }^{9}$ Meanwhile, the Phase II study of MK-2206 in recurrent or metastatic NPC was terminated due to limited activity. ${ }^{10}$ As shown in our results, AKT inactivation with small molecule inhibitors did not induce significant apoptosis, but markedly increased autophagy by regulating the mTOR and the S6 kinase pathway, which is a critical regulator of autophagy. ${ }^{9}$ Autophagy is characterized by the formation of double membrane vacuoles (autophagosome) in the cytoplasm, which support cell survival during metabolic stress and maintain normal homeostasis. ${ }^{11}$ However, in cancer, autophagy is considered to have dual functions as both a tumor suppressor and a protector of tumor cell survival. ${ }^{12}$ Increasing evidence shows that autophagy causes cell resistance to antineoplastic therapies; it has also been demonstrated to support cell survival. ${ }^{13}$ Thus, autophagy may serve as a mechanism by which the cell escapes therapyinduced cell death, and in these situations, the inhibition of autophagy may be a good therapeutic strategy. ${ }^{14}$

We hypothesized that the modest activity of MK2206 alone could be due to the presence of autophagy, which is induced by AKT inhibition. As a result, we conducted this study to investigate whether suppressing autophagy can enhance the effectiveness of MK-2206 against NPC.

\section{Materials and methods}

\section{Cell lines and culture}

NPC cell lines, including CNE-2 (Epstein-Barr virus negative) and C666-1 (Epstein-Barr virus positive), were supplied by the State Key Laboratory of Oncology in South China, People's Republic of China. Both cell lines were maintained in RPMI-1640 medium (Thermo Fisher Scientific, Waltham, MA, USA) containing 10,000 U/mL penicillin and $10 \mu \mathrm{g} / \mathrm{mL}$ streptomycin. The concentration of fetal bovine serum (Thermo Fisher Scientific) used in the medium was 10\% for CNE-2\% and 20\% for C666-1. Cells were incubated in a humidified atmosphere at $37^{\circ} \mathrm{C}$ with $5 \%$ carbon dioxide and $95 \%$ air.

\section{Reagents and antibodies}

The AKT inhibitor MK-2206 was obtained from Merck \& Co., Inc. (Whitehouse Station, NJ, USA). Bafilomycin A1 (Bafil A1), NH125 and hydroxychloroquine sulfate (HCQ) were purchased from Selleck Chemicals (Houston, TX, USA). The working stocks were dissolved to $10 \mathrm{mM}, 50 \mu \mathrm{M}$ and $1 \mathrm{mM}$ using dimethyl sulfoxide (DMSO) for MK-2206, Bafil A1 and NH125, respectively. All reagents were stored at $-20^{\circ} \mathrm{C}$ and diluted in fresh culture medium before experiments. The concentration of DMSO in the final solution did not exceed $1 \%(\mathrm{v} / \mathrm{v})$. The stock solution of HCQ was formulated to $50 \mathrm{mM}$ with ultrapure water. In vivo, 30\% Captisol (CyDex Pharmaceuticals, Inc., Lenexa, KS, USA) was used to dose the MK-2206. LC3 I/II, $\beta$-actin, phospho-eEF2 (Thr56), eEF2, eEF2K, SQSTM1/p62 (D5E2) and cleaved caspase-3 (Asp175) were obtained from Cell Signaling Technology (Danvers, MA, USA). Anti-hypoxia-inducible factor- $1 \alpha$ antibody was purchased from BD Biosciences (San Diego, CA, USA).

\section{RNA-mediated gene knockdown}

Tumor cells were seeded at a density of $8.0 \times 10^{4}$ cells/well for CNE-2 and at $1.6 \times 10^{5}$ cells/well for C666-1 in six-well plates, grown overnight and transfected with siRNA or shRNA or control. Eukaryotic elongation factor-2 (eEF-2) kinase siRNA (eEF-2K siRNA) and control siRNA (NT) were synthesized by Shanghai Gene-Pharma Co. (Shanghai, China). Lentivirus-based shRNA targeting eEF-2 kinase (eEF-2K shRNA) and nontargeting shRNA control (vector) were obtained from Genechem Co., Ltd. (Shanghai, China). Target sequence for eEF-2K siRNA was as follows: forward primer 5'-CCUGGAAGUGCAAAGGCUUTT-3' and reverse primer $5^{\prime}$-AAGCCUUUGCACUUCCAGGTT-3'. Detailed transfection steps were performed following the manufacturer's instructions.

\section{Western blot analysis}

Cells were lysed for 30 minutes in RIPA lysis buffer (Thermo Fisher Scientific), which contained protease (Sigma-Aldrich Co., St Louis, MO, USA) and phosphatase inhibitor cocktail PhosSTOP (Hoffman-La Roche Ltd., Basel, Switzerland). The lysates were centrifuged at 13,000 rpm for 20 minutes to collect the supernatant. The protein concentration was measured by a Thermo Protein Assay Reagent (Thermo Fisher Scientific). Next, 30-100 $\mu \mathrm{g}$ protein was loaded into a 6\%-15\% sodium dodecyl sulfate-polyacrylamide gel electrophoresis gel and electrophoretically transferred to polyvinylidene difluoride membranes (Bio-Rad Laboratories Inc., Hercules, CA, USA). The membranes were incubated overnight at $4^{\circ} \mathrm{C}$ with primary antibodies, followed by further incubation with secondary antibodies at room temperature for 1 hour. Bands were detected using super signal West Dura Luminol/Enhancer solution (Thermo Fisher Scientific) according to the manufacturer's protocol. 


\section{Cell viability analysis}

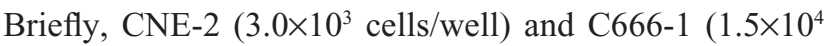
cells/well) were seeded in 96-well plates and incubated with different treatments for 72 hours. After 1-4 hours of incubation, the cell viability was evaluated using Cell Counting Kit-8 (CCK-8) (Dojindo Co., Kumamoto, Japan) according to the manufacturer's instructions. The viability of the control group was normalized to $100 \%$. Cell survival rate $(\%)=(\mathrm{OD}$ value of treatment group/OD value of control group) $\times 100 \%$. All experiments were performed in triplicate in at least three independent experiments.

\section{Animal study}

Male BALB/c nude mice weighing 15-18 g were subcutaneously inoculated with CNE- 2 cells $\left(5 \times 10^{6}\right.$ cells $/ 0.2 \mathrm{~mL} /$ injection). After inoculation, the mice whose tumor volume reached $\sim 100-200 \mathrm{~mm}^{3}$ were randomized into the following groups $^{15,16}$ (eight mice per group): 1) control; 2) MK-2206 alone; 3) NH125 alone and 4) combination of MK-2206 and NH125. MK-2206 (120 mg/kg, dissolved in 30\% Captisol, oral gavage three times a week), NH125 $(500 \mu \mathrm{g} / \mathrm{kg}$, dissolved in PBS with 2\% DMSO, intraperitoneal injection every day) and vehicle control (30\% Captisol $10 \mathrm{~mL} / \mathrm{kg}$ by oral gavage three times a week and PBS with $2 \%$ DMSO injected intraperitoneally daily) were administered for 2 weeks. Tumor growth was measured with calipers every other day, and the tumor volume was calculated by the following formula: volume $\left(\mathrm{mm}^{3}\right)=$ length $\times$ width $^{2} \times 0.5 .{ }^{16}$

\section{Statistical analysis}

All the in vitro experiments were repeated at least three times. The representative results were displayed as the mean value $\pm \mathrm{SD}$ of the mean. Student's $t$-test was used to compare the mean, and $P$-values $<0.05$ were considered statistically significant. Data were analyzed by GraphPad Prism 5 software (GraphPad Software Inc., La Jolla, CA, USA). Kolmogorov-Smirnov and Shapiro-Wilk tests were used to test normality of our data. When tumor volumes, body weights and tumor weights were normally distributed, comparisons for mean tumor volumes, mean body weights and mean tumor weights in the xenograft studies were analyzed by one-way analysis of variance, and $P$-values of pairwise comparisons were adjusted with Dunnett correction or Bonferroni correction. Two-sided $P<0.05$ was considered statistically significant. When data were not normally distributed, Kruskal-Wallis test was used to test the differences. We estimated that compared with the control group, tumor volume in the combined treatment group was reduced by $50 \%$ after treatment. Eight mice in each group would be required to achieve an $80 \%$ power to detect the difference at an alpha level of 0.05. Statistical analysis was performed using SPSS version 16.0 software (SPSS Inc., Chicago, IL, USA).

\section{Ethics}

All animal experiments were performed in accordance with the rules of the National Institutes of Health Guide for the Care and Use of Laboratory Animals and the UK Coordinating Committee on Cancer Research. The protocols of the animal experiments and the use of the cell lines were approved by the Animal Care and Use Committee of Sun Yat-sen University Cancer Center.

\section{Results}

MK-2206 induces an eEF-2 kinasedependent autophagy in NPC cells under both normoxic and hypoxic conditions

In a previous study, we found that the AKT inhibitor, MK-2206, did not clearly induce apoptosis, but caused autophagy in NPC cell lines as evidenced by an increase in the amount of LC3 II and double- or multi-membrane vacuoles in the cytoplasm when visualized by electron microscopy. ${ }^{9}$ As shown in Figure 1A and C, the effect of MK-2206 on autophagy was confirmed in this study. MK-2206 increased the level of LC3-II in a concentration-dependent manner in the human NPC cell lines CNE-2 and C666-1. Bafil A1 is an inhibitor of autophagosome-lysosome fusion and LC3 II degradation. ${ }^{17}$ When MK-2206 was combined with Bafil A1, the level of LC3 II was further enhanced, indicating that autophagic flux was enhanced by this AKT inhibitor (Figure 1A). Several lines of evidence suggest that eEF-2 kinase may be a regulator of autophagy. ${ }^{18}$ Therefore, we tested whether eEF-2 kinase was involved in autophagy induced by AKT inhibition. As shown in Figure 1B, MK-2206 activated eEF-2 kinase in a concentration-dependent manner by increasing the phosphorylation of eEF-2, the substrate for the enzyme. The results suggested that eEF-2K participated in the autophagy induced by MK-2206. The hypoxic microenvironments of solid tumors are believed to be linked to malignant features, including poor response. ${ }^{19}$ The effect of MK-2206 in NPC cells lines under hypoxia was also assessed. Hypoxia-inducible factor- $1 \alpha$ expression was measured to confirm hypoxia by Western blotting (Figure 1C). The Western blot results demonstrated that MK-2206 induced a stronger autophagic response and higher eEF-2 kinase activity under a hypoxic condition than under a normoxic condition (Figure 1C). 
A

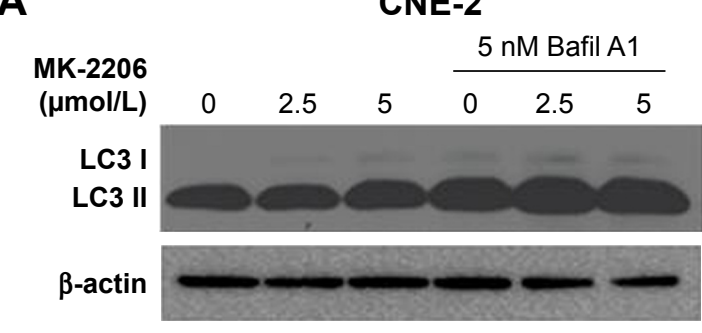

B

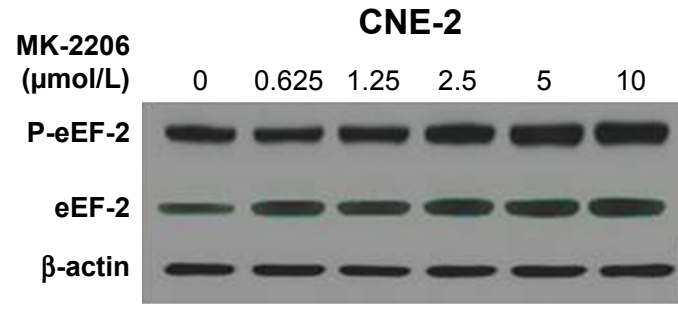

C

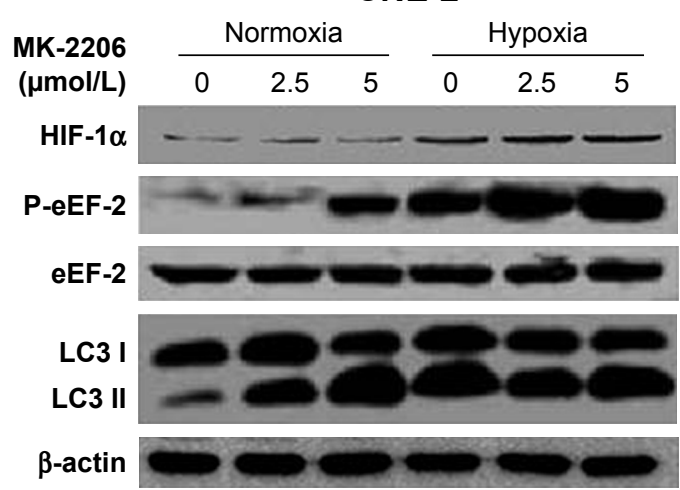

C666-1

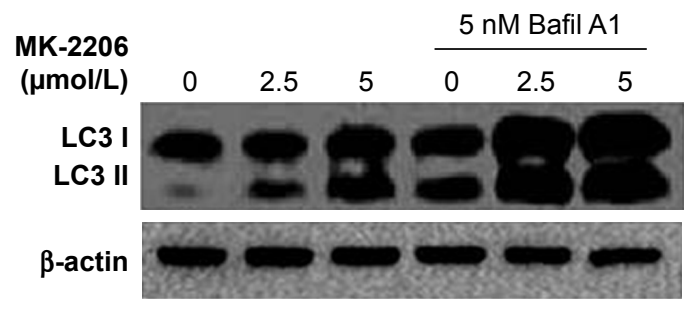

C666-1

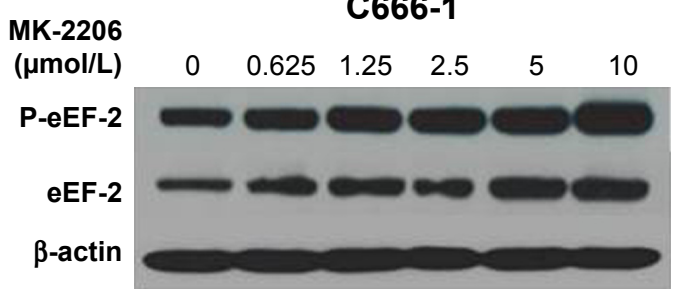

C666-1

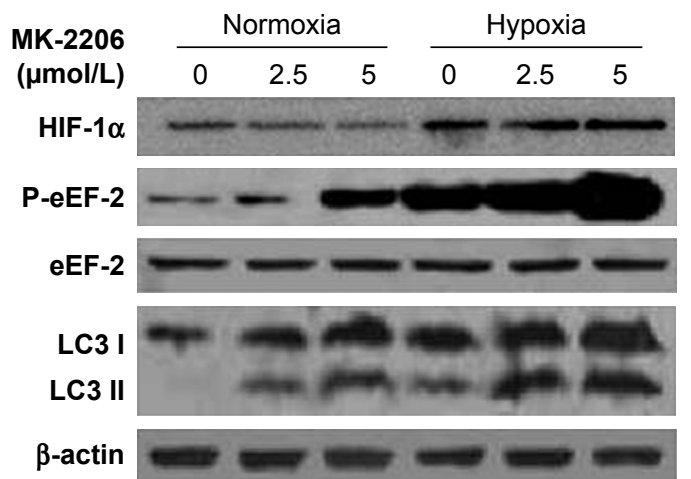

Figure I Effects of MK-2206 on autophagy in human NPC cell lines.

Notes: (A) CNE-2 and C666-I cells cultured in medium supplemented with 10\% and 20\% FBS were treated with MK-2206 for 24 hours in the absence or presence of 5 nM (nmol/L) Bafil AI, and the level of LC3 was examined by Western blotting. (B) CNE-2 and C666-I cells were treated with a series of concentrations of MK-2206 for 24 hours, and P-eEF-2 and eEF-2 were examined by Western blotting. (C) CNE-2 and C666-I cells were treated with 2.5 and 5 mmol/L MK-2206 under normoxia or hypoxia $\left(\mathrm{I} \% \mathrm{O}_{2}\right)$ for 24 hours. HIF- Ia, P-eEF-2, eEF-2 and LC3 were examined by Western blotting. $\beta$-Actin was used as a loading control.

Abbreviations: Bafil, bafilomycin; FBS, fetal bovine serum; HIF, hypoxia-inducible factor; eEF-2, eukaryotic elongation factor-2; NPC, nasopharyngeal carcinoma; P-eEF-2, phospho-eEF-2.

\section{Silencing of eEF-2 kinase expression blunts autophagy activated by MK-2206 in NPC cell lines}

To further verify whether eEF-2 kinase plays a regulatory role in MK-2206-induced autophagy in NPC cells, we applied RNA interference techniques to specifically and stably knock down eEF-2 kinase using shRNA in CNE-2 and C666-1 cells. Figure 2A shows that compared with cells without silencing of the kinase, NPC cells transfected with an eEF-2 kinase shRNA exhibited decreased autophagy, with a considerable decrease in LC3-II and increases in p62/sequestosome1 (SQSTM1) protein levels in Western blot analysis. These results indicate that autophagic response to MK-2206 treatment was blunted by inactivation of eEF-2 kinase and further support a role for eEF-2 kinase in activating autophagy in NPC cells. Under hypoxic condition, knockdown of eEF-2 kinase blunted autophagy activated by MK-2206 and hypoxia (Figure 2B).

\section{Suppression of autophagy via inhibiting eEF-2 kinase enhances the cytocidal effect of MK-2206 in human NPC cells}

To further test our hypothesis that autophagy plays a prosurvival role in response to AKT inhibitors, we knocked down eEF-2 kinase via shRNA in the CNE-2 and C666-1 cell lines. After treatment with a series of concentrations of MK-2206, we compared the growth and survival of these autophagydeficient cells with those of the cells transfected with a nontargeting RNA using a CCK-8 colorimetric assay. As shown in Figure 3A, the suppression of autophagy via knockdown of eEF-2 kinase genes caused more death together with MK-2206 in the NPC cell lines. NH125 is a potent and relatively specific inhibitor of eEF-2 kinase. ${ }^{20} \mathrm{We}$ observed that 
A

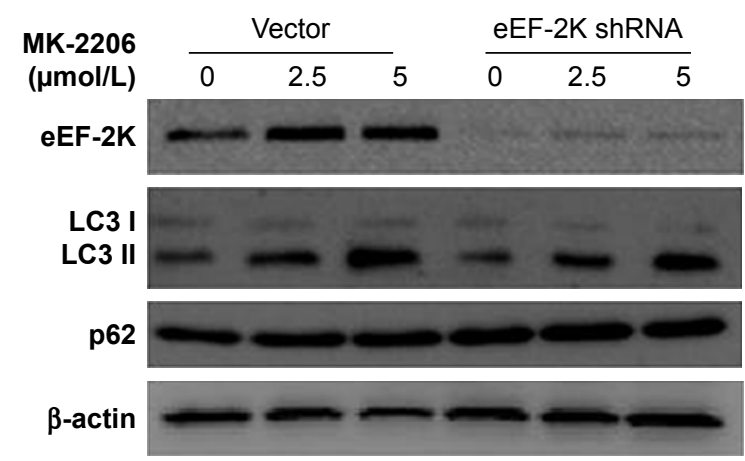

B

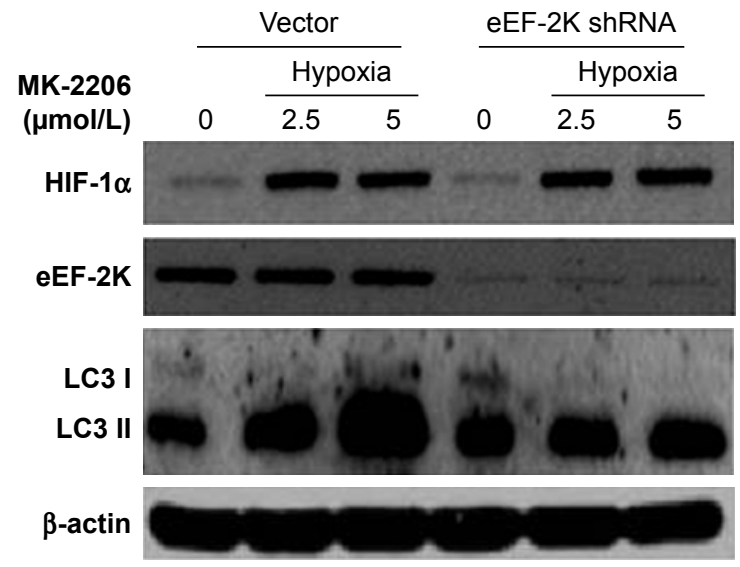

C666-1

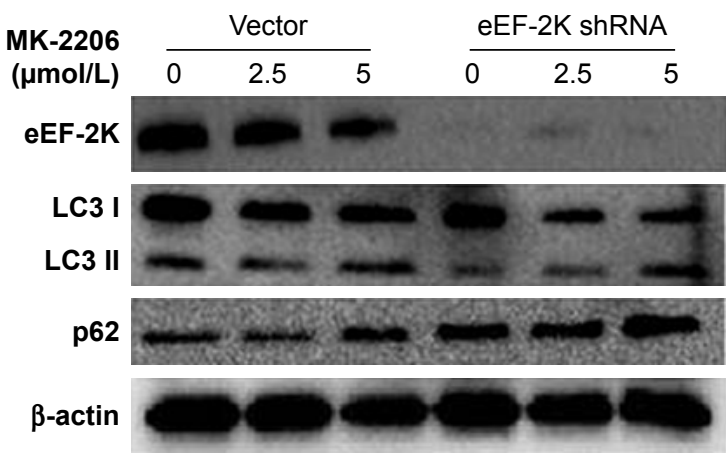

C666-1

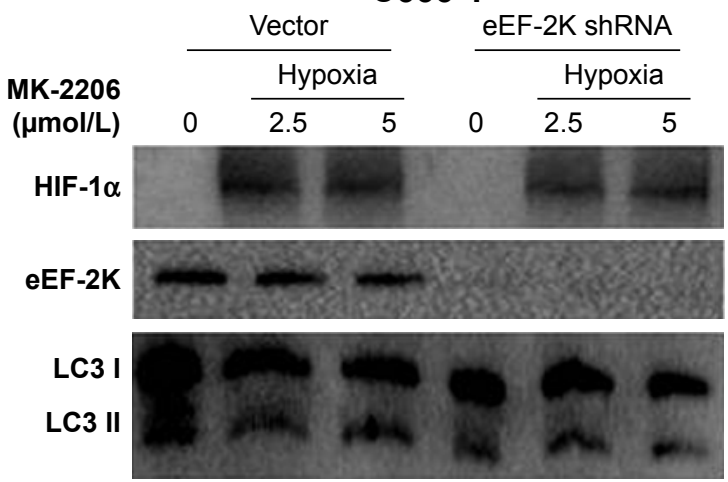

$\beta$-actin

Figure 2 AKT inhibition induced eEF-2 kinase-dependent autophagy.

Notes: (A) CNE-2 and C666-I cells were transfected with a nontargeting RNA (Vector) or an shRNA targeting eEF-2 kinase (eEF-2K shRNA), followed by treatment with MK-2206 for 24 hours. eEF-2 kinase, LC3 and p62 were examined by Western blotting. $\beta$-Actin was used as a loading control. (B) CNE- 2 and C666-I cells were transfected with a nontargeting RNA or an eEF-2 kinase-targeted shRNA, followed by treatment with MK-2206 for 24 hours under hypoxia (I\% $\mathrm{O}_{2}$ ). HIF-I $\alpha$, eEF-2 kinase and LC3 were examined by Western blotting. $\beta$-Actin was used as a loading control.

Abbreviation: HIF, hypoxia-inducible factor; eEF-2, eukaryotic elongation factor- 2 .

compared with treatment with MK-2206 alone, the combined treatment of MK-2206 with NH125 significantly increased the growth-inhibitory effect of MK-2206 on the human NPC cell lines CNE-2 and C666-1 (Figure 3B). Inhibition of eEF-2 kinase using the eEF-2 kinase-targeted shRNA or NH125 sensitized NPC cancer cells to the AKT inhibitor.

HCQ, a drug with a favorable and well-defined toxicity profile, is commonly used to treat autoimmune diseases
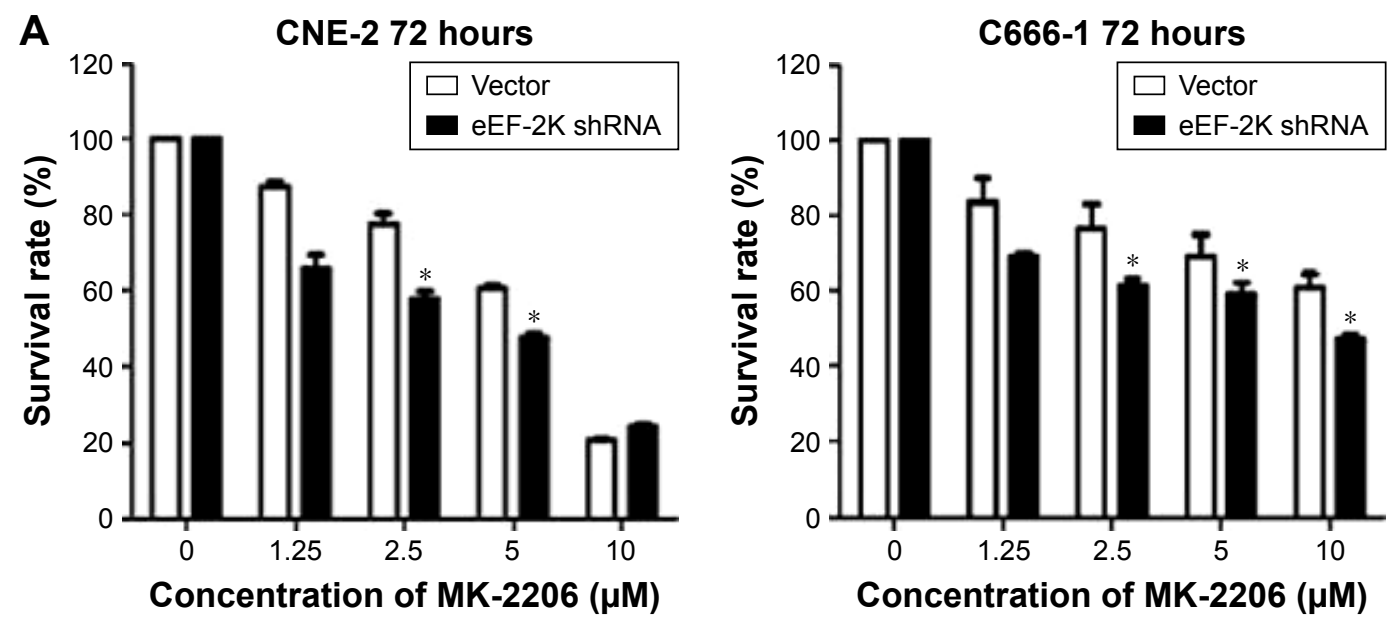

Figure 3 (Continued) 

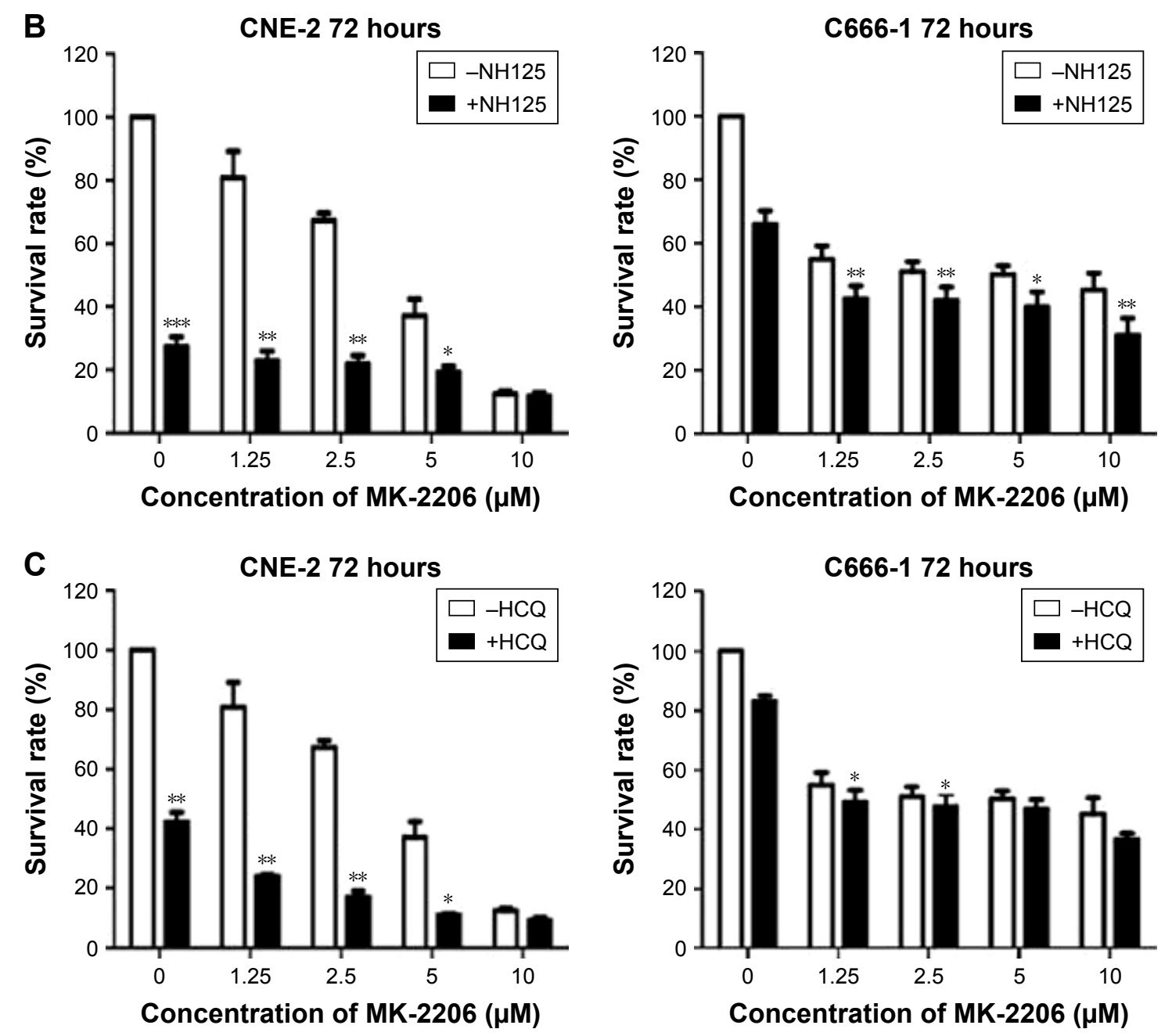

D

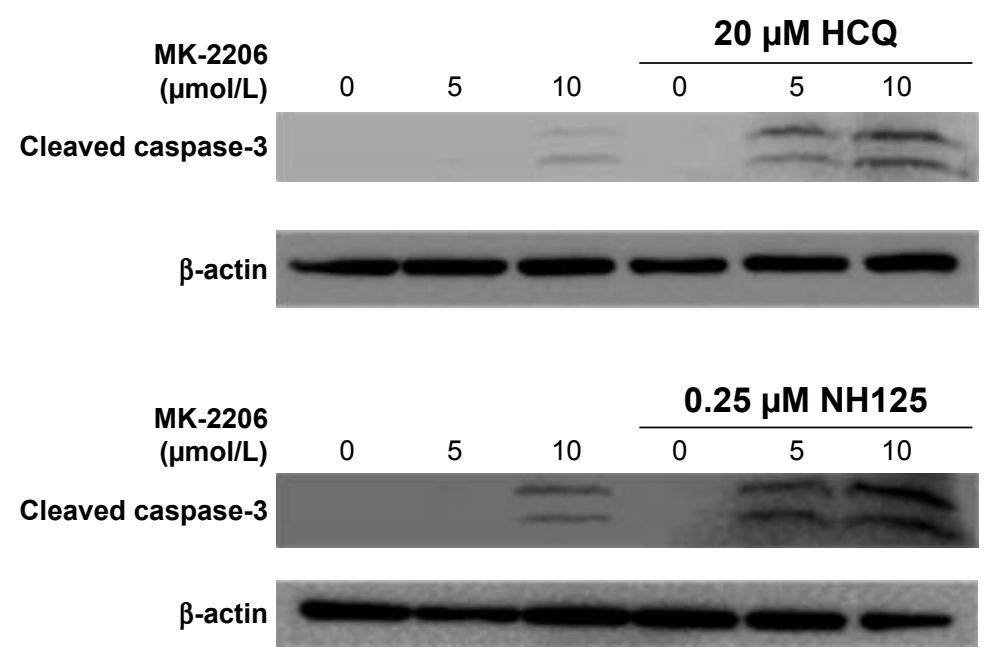

Figure 3 Suppression of autophagy increases the cytocidal activity of MK-2206 in NPC cell lines.

Notes: (A) CNE-2 and C666-I cells were transfected with a nontargeting RNA or an shRNA targeting eEF-2 kinase (eEF-2K shRNA), followed by treatment with a series of concentrations of MK-2206 for 72 hours in fully supplemented medium. (B) CNE-2 and C666-I cells cultured in medium supplemented with $10 \%$ FBS and $20 \%$ FBS were treated with a series of concentrations of MK-2206 for 72 hours in the presence or absence of $0.25 \mu \mathrm{M} \mathrm{NHI25}$. (C) CNE-2 and C666-I cells cultured in medium supplemented with 10\% FBS and $20 \%$ FBS were treated with a series of concentrations of MK-2206 for 72 hours in the presence or absence of $20 \mu \mathrm{M}$ HCQ. At the end of treatment, cell viability was measured via the CCK-8 assay. The results shown represent the mean \pm SD of quadruplicate determinations from one of three identical experiments; $* P<0.05, * * P<0.01$, $* * * P<0.00$ I. (D) CNE-2 cultured in medium supplemented with $10 \%$ FBS was treated with a series of concentrations of MK-2206 for 72 hours in the presence or absence of $20 \mu \mathrm{M} \mathrm{HCQ}$ and $0.25 \mu \mathrm{M} \mathrm{NHI} 25$. Cleaved caspase- 3 was measured by Western blotting, and $\beta$-actin was used as a loading control. Abbreviations: CCK-8, Cell Counting Kit-8; FBS, fetal bovine serum; HCQ, hydroxychloroquine; NPC, nasopharyngeal carcinoma; eEF-2, eukaryotic elongation factor-2. 
and is a known inhibitor of autophagy. ${ }^{21}$ We used HCQ as a control, and the results showed that HCQ increased the sensitivity of the NPC cell lines to MK-2206, similar to that with the eEF-2 kinase inhibitor (Figure 3C). Inhibition of autophagy using an autophagy inhibitor also increased MK-2206-induced cell death.

Western blot analysis was subsequently performed to analyze the levels of cleaved caspase-3, a marker of apoptosis in NPC cell lines. There was an increase in the level of cleaved caspase- 3 in cells treated with the combination treatment, suggesting an increase of apoptosis in NPC cell lines (Figure 3D).

\section{Effect of the combined treatment with MK- 2206 and NHI 25 on NPC growth in vivo}

In our previous study, using an NPC xenograft model with CNE2 cells, we observed that MK-2206 alone can inhibit tumor growth, and the dose of MK-2206 in the present study was determined according to the previous results. ${ }^{9}$ Here, we evaluated the therapeutic benefit of the combined treatment of MK-2206 with the eEF-2 kinase inhibitor NH125 in CNE-2 xenograft in nude mice. MK-2206 was dosed with $30 \%$ Captisol. The tumor-bearing mice were treated with the vehicle, MK-2206 or NH125 alone, and a combination of MK-2206 and NH125. Figure 4A and D shows that compared with the vehicle, MK-2206 alone exhibited tumor-inhibitory effects. However, the combination of MK-2206 and NH125 caused a greater inhibition of tumor growth than did MK-2206 alone. At the end of the experiment, we obtained identical results by directly measuring tumor weights. The tumor weights were much lighter in the MK-2206 and NH125 combination group than in the other three groups $(P<0.05$; Figure 4B). Body weight reduction was observed in the MK-2206 and NH125 co-administration group (Figure 4C). Tumor volumes, body weights and tumor weights were normally distributed; statistical differences were tested by

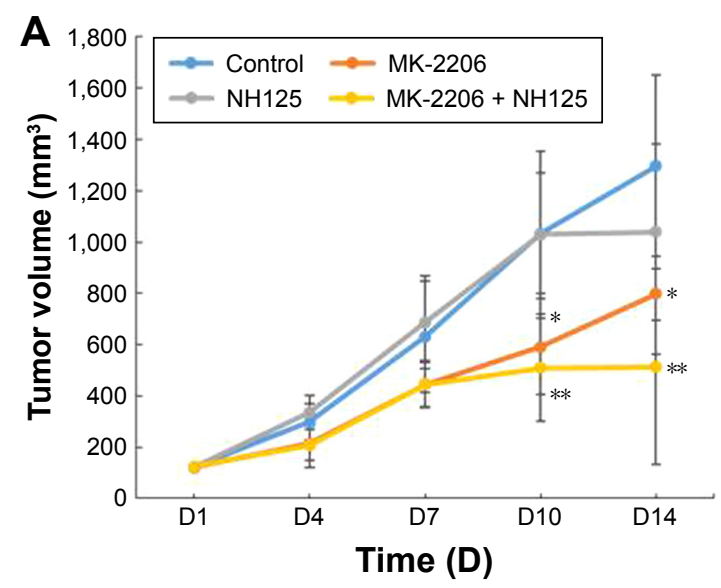

C
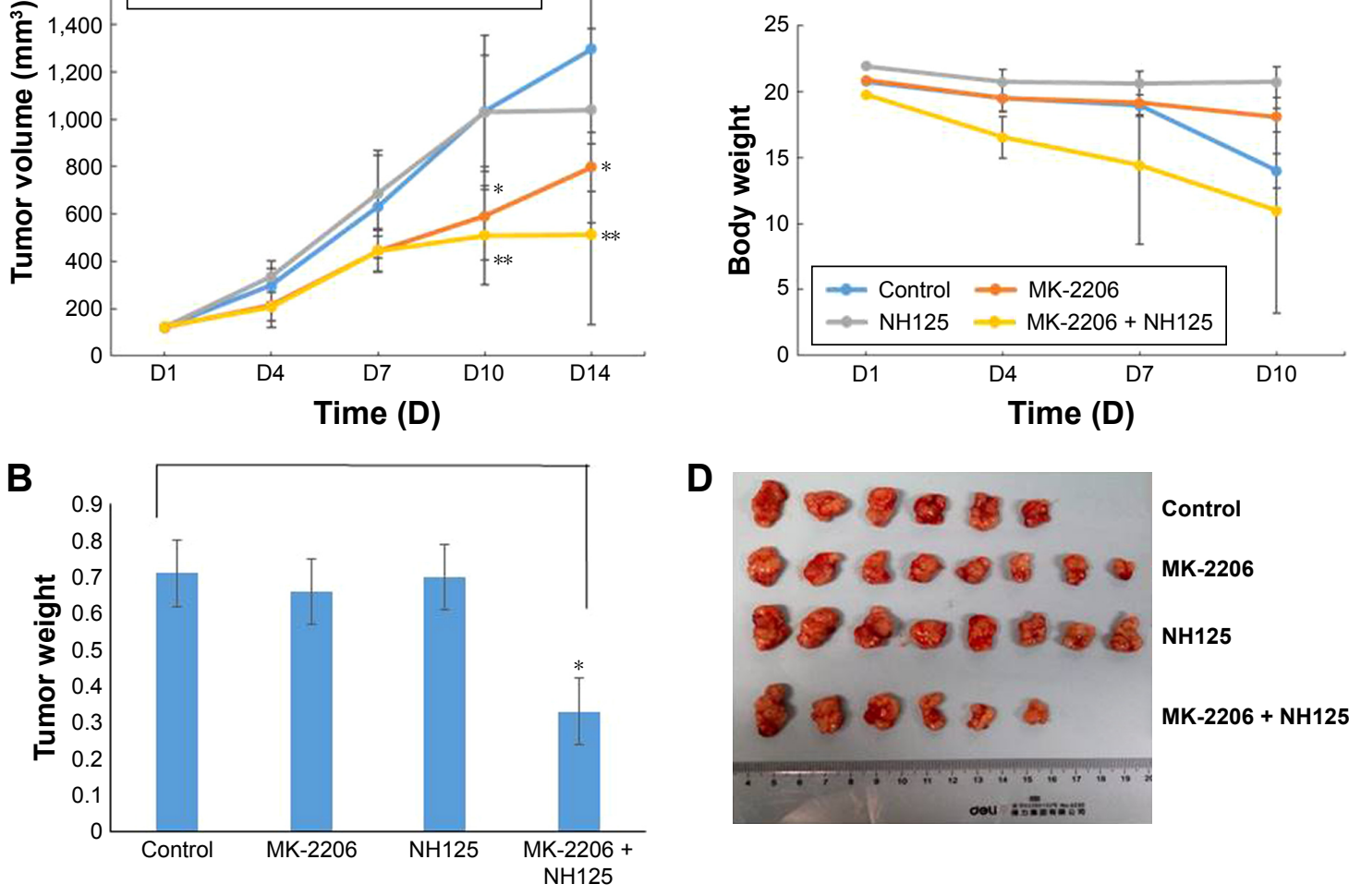

D

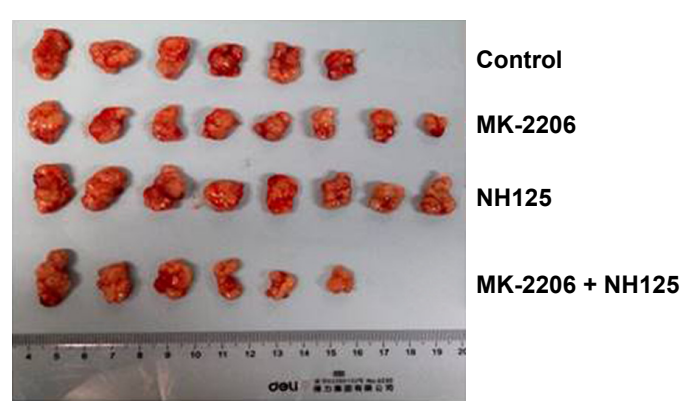

Figure 4 Combined treatment with MK-2206 and NHI 25 suppressed the growth of human CNE-2 xenografts in nude mice.

Notes: The tumor-bearing mice were divided into four groups: I) control; 2) MK-2206 alone; 3) NHI25 alone and 4) combination of MK-2206 and NHI25. The treatments began on day I after grouping and continued for 2 weeks. MK-2206 (120 mg/kg, dissolved in 30\% Captisol) three times a week by oral gavage; NHI 25 (500 $\mu$ g/kg, dissolved in PBS with $2 \%$ DMSO) injected intraperitoneally daily; vehicle for control group (30\% Captisol $10 \mathrm{~mL} / \mathrm{kg}$ by oral gavage three times a week and PBS with $2 \%$ DMSO injected intraperitoneally daily). Points: mean of tumor volume; bars: SD. $* P<0.05$ compared with vehicle control; $* * P<0.01$ compared with vehicle control. (A) Tumor volume, (B) tumor weight, $(\mathbf{C})$ body weight and $(\mathbf{D})$ images of the tumors.

Abbreviation: DMSO, dimethyl sulfoxide. 
one-way analysis of variance. Two mice died in the control and the combination groups, respectively, during the treatment. No other obvious toxicity was observed in the mice.

\section{Discussion}

Our previous study demonstrated that an AKT inhibitor (MK-2206) induced G0/G1 cycle arrest without significant apoptosis in NPC, which is in accordance with another study reported by Ma et al., ${ }^{9,22}$ Additionally, we showed that the activity of the TSC2/mTOR/S6 kinase pathway was inhibited following AKT inhibition. ${ }^{9}$ Since this pathway is a known inhibitor of autophagy, we hypothesized that its inhibition may induce autophagy. This hypothesis was confirmed in our previous study and in the present study (Figure 1). AKT inhibition can induce autophagy in NPC, which is similar to previous studies reporting that AKT knockdown or inactivation with small molecule inhibitors did not induce significant apoptosis, but markedly increased autophagy. ${ }^{23}$ eEF-2 kinase, also known as calmodulin-dependent protein kinase III, is a unique calcium/calmodulin-dependent enzyme that regulates protein synthesis. ${ }^{24}$ The only known substrate of this kinase is eEF-2, and eEF-2 kinase phosphorylates eEF-2 on Thr56. ${ }^{24}$ eEF-2 kinase can act as a positive regulator of autophagy under environmental or metabolic stress, ${ }^{25}$ the activity of which was reported to be controlled via the mTOR/S6 kinase pathway. ${ }^{26}$ Consequently, in this study, we sought to determine whether eEF-2 kinase was involved in the autophagy induced by AKT inhibition. Figure $1 \mathrm{~B}$ and C shows that eEF-2 kinase was activated and that phosphorylation of eEF-2 was increased following the induction of autophagy triggered by AKT inhibitor.

Next, we aimed to determine if inhibition of eEF-2 kinase can inhibit autophagy caused by AKT inhibitors. Our results showed that inactivation of eEF-2 kinase blunted autophagy activated by MK-2206 and hypoxia (Figure 2). In cancer, increasing evidence shows that autophagy causes cell resistance to antineoplastic therapies. In these situations, the inhibition of autophagy may be a good therapeutic strategy. ${ }^{14}$ Autophagy inhibition via chloroquine derivatives can enhance the cytotoxicity of several chemotherapies and targeted therapies. ${ }^{27}$ Phase I clinical trials evaluating HCQ as an autophagy inhibitor in combination with cytotoxic chemotherapy ${ }^{28,29}$ and cell signaling inhibitors ${ }^{30}$ have shown encouraging early results. Therefore, in our study, we further investigated whether autophagy suppression can increase the sensitivity of MK-2206. As shown in Figure 3, using the eEF-2 kinasetargeted shRNA or NH125 sensitized the NPC cancer cells to the AKT inhibitor in vitro. The synergistic effect is similar to the effect with HCQ combined with the AKT inhibitor. In the NPC xenograft model with CNE2 cells, NH125 showed good synergistic effects with MK-2206 in vivo (Figure 4). All these results demonstrated that blocking autophagy could enhance the sensitivity of NPC to AKT inhibitors.

More recently, it was reported that inhibition of eEF-2 kinase sensitizes human NPC cell lines to lapatinib. ${ }^{31}$ In breast cancer, suppression of eEF-2 kinase-regulated autophagy augments the efficacy of growth factor inhibitors such as trastuzumab, gefitinib and lapatinib. ${ }^{32}$ The results of all these studies suggested that activation of eEF-2 kinase-mediated autophagy played a protective role in cancer cells, and that targeted autophagic survival may enhance the effectiveness of growth factor inhibitors.

In summary, the results of this study provide experimental evidence that the eEF-2 kinase-mediated autophagy induced by AKT inhibition plays a protective role in NPC cells. Targeting autophagic survival may represent a novel approach to enhance the effectiveness of growth factor inhibitors. These findings also suggest that inhibition of eEF-2 kinase may be an effective method for increasing the efficacy of AKT inhibitors such as MK-2206 in NPC.

\section{Acknowledgment}

This work was funded by the China National Natural Science Foundation (81502352).

\section{Disclosure}

The authors report no conflicts of interest in this work.

\section{References}

1. Torre LA, Bray F, Siegel RL, et al. Global cancer statistics, 2012. $C A$ Cancer J Clin. 2015;65(2):87-108.

2. Cao SM, Simons MJ, Qian CN. The prevalence and prevention of nasopharyngeal carcinoma in China. Chin J Cancer. 2011;30(2):114-119.

3. Bhattacharyya $\mathrm{N}$. The impact of race on survival in nasopharyngeal carcinoma: a matched analysis. Am J Otolaryngol. 2004;25(2):94-97.

4. Tan W-L, Tan E-H, Lim DW-T, et al. Advances in systemic treatment for nasopharyngeal carcinoma. Chin Clin Oncol. 2016;5(2):21.

5. Yu KH, Leung SF, Tung SY, et al. Survival outcome of patients with nasopharyngeal carcinoma with first local failure: a study by the Hong Kong Nasopharyngeal Carcinoma Study Group. Head Neck. 2005;27(5):397-405.

6. Manning BD, Cantley LC. AKT/PKB signaling: navigating downstream. Cell. 2007;129(7):1261-1274.

7. Cheung AK, Ip JC, Chu AC, et al. PTPRG suppresses tumor growth and invasion via inhibition of Akt signaling in nasopharyngeal carcinoma. Oncotarget. 2015;6(15):13434-13447.

8. Wang W, Wen Q, Xu L, et al. Activation of Akt/mTOR pathway is associated with poor prognosis of nasopharyngeal carcinoma. PLoS One. 2014;9(8):e106098.

9. Zhao YY, Tian Y, Zhang J, et al. Effects of an oral allosteric AKT inhibitor (MK-2206) on human nasopharyngeal cancer in vitro and in vivo. Drug Des Devel Ther. 2014;8:1827-1837. 
10. Ma BB, Goh BC, Lim WT, et al. Multicenter Phase II study of the AKT inhibitor MK-2206 in recurrent or metastatic nasopharyngeal carcinoma from patients in the Mayo Phase II consortium and the cancer therapeutics research group (MC1079). Invest New Drugs. 2015;33(4): 985-991.

11. Choi AM, Ryter SW, Levine B. Autophagy in human health and disease. N Engl J Med. 2013;368(19):651-662.

12. White E, Dipaola RS. The double-edged sword of autophagy modulation in cancer. Clin Cancer Res. 2009;15(17):5308-5316.

13. Kundu M, Thompson CB. Macroautophagy versus mitochondrial autophagy: a question of fate? Cell Death Differ. 2005;12(Suppl 2): 1484-1489.

14. Wang Z, Han W, Sui X, Fang Y, Pan H. Autophagy: a novel therapeutic target for hepatocarcinoma (review). Oncol Lett. 2014;7(5): 1345-1351.

15. Hirai H, Sootome H, Nakatsuru Y, et al. MK-2206, an allosteric Akt inhibitor, enhances antitumor efficacy by standard chemotherapeutic agents or molecular targeted drugs in vitro and in vivo. Mol Cancer Ther. 2010;9(7):1956-1967.

16. Chiong E, Lee IL, Dadbin A, et al. Effects of mTOR inhibitor everolimus (RAD001) on bladder cancer cells. Clin Cancer Res. 2011;17(9): 2863-2873.

17. Klionsky DJ, Elazar Z, Seglen PO, Rubinsztein DC. Does bafilomycin A1 block the fusion of autophagosomes with lysosomes? Autophagy. 2008;4(7):849-850.

18. Hait WN, Wu H, Jin S, Yang JM. Elongation factor-2 kinase: its role in protein synthesis and autophagy. Autophagy. 2006;2(4):294-296.

19. Matsumoto S, Yasui H, Mitchell JB, Krishna MC. Imaging cycling tumor hypoxia. Cancer Res. 2010;70(24):10019-10023.

20. Arora S, Yang JM, Kinzy TG, et al. Identification and characterization of an inhibitor of eukaryotic elongation factor 2 kinase against human cancer cell lines. Cancer Res. 2003;63(20):6894-6899.

21. Amaravadi RK, Yu D, Lum JJ, et al. Autophagy inhibition enhances therapy-induced apoptosis in a Myc-induced model of lymphoma. J Clin Invest. 2007;117(2):326-336.

22. Ma BB, Lui VW, Hui CW, et al. Preclinical evaluation of the AKT inhibitor MK-2206 in nasopharyngeal carcinoma cell lines. Invest New Drugs. 2013;31(3):567-575.
23. Degtyarev $\mathrm{M}$, de Mazière A, Orr C, et al. Akt inhibition promotes autophagy and sensitizes PTEN-null tumors to lysosomotropic agents. $J$ Cell Biol. 2008;183(1):101-116.

24. Ryazanov AG, Shestakova EA, Natapov PG. Phosphorylation of elongation factor 2 by EF-2 kinase affects rate of translation. Nature. 1988; 334(6178):170-173

25. Py BF, Boyce M, Yuan J. A critical role of eEF-2K in mediating autophagy in response to multiple cellular stresses. Autophagy. 2009;5(3): 393-396.

26. Browne GJ, Proud CG. A novel mTOR-regulated phosphorylation site in elongation factor 2 kinase modulates the activity of the kinase and its binding to calmodulin. Mol Cell Biol. 2004;24(7): 2986-2997.

27. Amaravadi RK, Lippincott-Schwartz J, Yin XM, et al. Principles and current strategies for targeting autophagy for cancer treatment. Clin Cancer Res. 2011;17(4):654-666.

28. Rangwala R, Leone R, Chang YC, et al. Phase I trial of hydroxychloroquine with dose-intense temozolomide in patients with advanced solid tumors and melanoma. Autophagy. 2014;10(8):1369-1379.

29. Rosenfeld MR, Ye X, Supko JG, et al. A Phase I/II trial of hydroxychloroquine in conjunction with radiation therapy and concurrent and adjuvant temozolomide in patients with newly diagnosed glioblastoma multiforme. Autophagy. 2014;10(8):1359-1368.

30. Rangwala R, Chang YC, Hu J, et al. Combined MTOR and autophagy inhibition: Phase I trial of hydroxychloroquine and temsirolimus in patients with advanced solid tumors and melanoma. Autophagy.10(8): 1391-1402.

31. Liu L, Huang P, Wang Z, et al. Inhibition of eEF-2 kinase sensitizes human nasopharyngeal carcinoma cells to lapatinib-induced apoptosis through the Src and Erk pathways. BMC Cancer. 2016;16(1):813.

32. Cheng Y, Li H, Ren X, Niu T, Hait WN, Yang J. Cytoprotective effect of the elongation factor-2 kinase-mediated autophagy in breast cancer cells subjected to growth factor inhibition. PLoS One. 2010; 5(3):e9715.

\section{Publish your work in this journal}

Drug Design, Development and Therapy is an international, peerreviewed open-access journal that spans the spectrum of drug design and development through to clinical applications. Clinical outcomes, patient safety, and programs for the development and effective, safe, and sustained use of medicines are the features of the journal, which has also been accepted for indexing on PubMed Central. The manuscript management system is completely online and includes a very quick and fair peer-review system, which is all easy to use. Visit http://www.dovepress.com/testimonials.php to read real quotes from published authors. 